\title{
Nrf-2 signaling inhibits intracranial aneurysm formation and progression by modulating vascular smooth muscle cell phenotype and function
}

Yuan Shi ${ }^{1+}$, Sichen $\mathrm{Li}^{1+}$, Yaying Song ${ }^{2}$, Peixi Liu', Zixiao Yang ${ }^{1}$, Yingjun Liu', Kai Quan ${ }^{1}$, Guo Yu', Zhiyuan Fan ${ }^{1}$ and Wei Zhu ${ }^{1 *}$

\begin{abstract}
Background: Oxidative stress and vascular smooth muscle cell (VSMC) phenotypic modulation influence intracranial aneurysm (IA) formation and progression. Oxidative stress plays an important role in phenotype switching, and nuclear factor erythroid 2-related factor 2 (Nrf-2) is one of the main antioxidant systems. Unfortunately, little is known about how Nrf-2 signaling influences VSMC phenotype switches during IA pathogenesis.

Methods: We examined the effect of Nrf-2 activation IA on formation and progression in an elastase-induced rat IA model. We also developed a hydrogen peroxide $\left(\mathrm{H}_{2} \mathrm{O}_{2}\right)$-induced VSMC oxidative damage model. Then, we analyzed VSMC phenotype changes in the setting of Nrf-2 activation or inhibition in vitro. The proliferation, migration ability, and apoptosis rate of VSMCs were tested. Lastly, we measured the expression levels of antioxidant enzymes and inflammatory cytokines downstream of Nrf-2.

Results: Nrf-2 activation suppressed IA formation and progression in vivo. We confirmed Nrf-2 nuclear translocation and a VSMC switch from the contractile to synthetic phenotype. Nrf-2 activation inhibited the proliferation, migratory ability, and apoptosis rate enhanced by $\mathrm{H}_{2} \mathrm{O}_{2}$. Quantitative real-time polymerase chain reaction (PCR) and western blot analysis revealed that Nrf-2 activation promoted antioxidant enzymes and VSMC-specific marker gene expressions but decreased pro-inflammatory cytokine levels.

Conclusion: These results suggest that Nrf-2 exerts protective effects against IA development by preventing VSMCS from changing to a synthetic phenotype.
\end{abstract}

Keywords: Intracranial aneurysm, Nrf-2, Vascular smooth muscle cells phenotype, Oxidative stress, Inflammation

\section{Background}

Intracranial aneurysms (IAs) are pathological dilations at main bifurcations of cerebral arteries and are the most common cause of subarachnoid hemorrhage. Nearly 3$5 \%$ of the adult population carries unruptured IA [1]. The mechanisms of IA development and rupture remain unclear. Many factors such as blood flow wall shear stress, inflammation, oxidative stress, and apoptosis are involved

\footnotetext{
* Correspondence: drweizhu@fudan.edu.cn; drzhuwei@fudan.edu.cn †Yuan Shi and Sichen Li contributed equally to this work. 'Department of Neurosurgery, Huashan Hospital, Fudan University, 12 Wulumiqi Rd., Shanghai 200040, People's Republic of China Full list of author information is available at the end of the article
}

in IA etiology [2]. The abnormal hemodynamic changes that can lead to endothelial cell dysfunction are now considered to be the initiating factor [3]. Several inflammatory mediators are triggered and then release large amounts of inflammatory cytokines and oxidative factors. This can cause a number of pathological processes including disruption of internal elastic laminate, vascular smooth muscle cell (VSMC) phenotype modulation, and dysfunctional extracellular matrix (ECM) remodeling [2-4]. When vessel walls degenerate, intracranial arteries become too weak to resist the blood dynamic force, and pouchlike dilatations of cerebral arteries occur.

(c) The Author(s). 2019 Open Access This article is distributed under the terms of the Creative Commons Attribution 4.0 International License (http://creativecommons.org/licenses/by/4.0/), which permits unrestricted use, distribution, and 
VSMCs are a major cell type in vessel walls that carry out various functions. There are two different types of VSMCs: contractile and synthetic. Markers of contractile phenotype include smooth muscle 22 alpha (SM22 $\alpha$ ), smooth muscle alpha actin ( $\alpha \mathrm{SMA}), \mathrm{SM}$ myosin heavy chain (MHC), h1-calponin, and smoothelin [5]. In response to pathologic stimuli such as inflammation and oxidative stress, contractile phenotype VSMCs can convert to the synthetic type; this phenotype modulation is associated with deceased expression of contractile genes [6]. During these changes, VSMCs lose their ability to contract but contribute to recruitment of pro-inflammatory cells and remodeling of the vessel wall ECM [6]. Switching to synthetic type VSMCs is hypothesized to play an important role in several cardiovascular diseases [7].

Oxidative stress is a key contributor to IA formation and rupture [8]. Oxidative damage caused by reactive oxygen species (ROS) can injure vessel walls by generating unstable free radicals and recruiting pro-inflammatory cells [5]. ROS also play an essential role in inflammatory disorders and VSMC phenotype modulation [9]. Nuclear factor erythroid 2-related factor 2 (Nrf-2), which belongs to the CNC (cap ' $n$ ' collar) family of transcription factors, is one of the main endogenous antioxidant systems. In stress conditions, Nrf-2 translocates from the cytoplasm to the nucleus where it regulates the expression of antioxidant and anti-inflammatory genes [10]. However, little is known about the significance of Nrf-2 in IA pathophysiology. In the current study, we tested the hypothesis that activation of Nrf-2 signaling can inhibit IA progression by modulating VSMC phenotype and function.

\section{Methods}

\section{Patients and tissue samples}

The Institutional Review Board of the Huashan Hospital of Fudan University approved this study. Informed consent was obtained from all patients. IA and superficial temporal artery (STA) samples were obtained during surgery. All specimens were fixed in $10 \%$ formaldehyde and embedded in paraffin.

\section{Rat IA model}

All animal procedures were carried out according to the protocol of our Institutional Animal Care and Use Committee. The experimental protocol was reviewed and approved by the Ethics Committee of the Huashan Hospital affiliated with Fudan University in Shanghai, People's Republic of China. Male adult Sprague Dawley rats (body weight 200$220 \mathrm{~g}$; Jiesijie, China) were divided into two groups $(n=10)$. IAs were induced as described previously [11]. Briefly, the right common carotid artery of rats was ligated, and $10 \mu \mathrm{L}$ of $10 \mathrm{U} / \mathrm{mL}$ elastase was stereotactically injected into the basal cisterns through a small burr hole made $1.2 \mathrm{~mm}$ rostral and $0.7 \mathrm{~mm}$ lateral to the right of the bregma. The rats were anesthetized with $3 \%$ isoflurane throughout the procedure. All rats were fed a hypertensive diet after the surgery, and one group was treated with the Nrf-2 agonist tertbutylhydroquinone (tBHQ, MedChemExpress, NJ, USA) 50 $\mathrm{mg} / \mathrm{kg} / \mathrm{d}$ by gavage. After 30 days, rats were perfused with phosphate-buffered saline (PBS) and 4\% paraformaldehyde (PFA) under deep anesthesia, then infused with bromophenol blue solution. Aneurysms were defined as an outpouching of weakened vessel walls, the diameters of which were $150 \%$ greater than the patent artery. All samples were processed immediately as described above for human specimens.

\section{Hematoxylin and eosin (HE) staining and immunohistochemistry}

Tissues were cut into $5-\mu \mathrm{M}$ sections and placed on polylysine-coated slides prior to routine HE staining. For immunohistochemical analysis, anti-Nrf-2 (ab28947, Abcam, Cambridge, UK) (ab28947, Abcam, Cambridge, UK) was used for primary antibodies, and 3,3'-diaminobenzidine (DAB) plus chromogen (Thermo Fisher Scientific, Waltham, MA) was used for substrate visualization, according to the manufacturers' protocols.

\section{Immunofluorescence staining}

Five-micrometer-thick sections were incubated at $4{ }^{\circ} \mathrm{C}$ overnight with anti- SM22 $\alpha$ (1:400 dilution) and anti- $\alpha$ SMA (1: 200 dilution) (Abcam, Cambridge, UK). They were then incubated with appropriate fluorescence-labeled secondary antibodies (1:1000 dilution) for $2 \mathrm{~h}$ at room temperature. $\mathrm{Nu}$ clei were stained with DAPI (1:1000 dilution). All images were obtained with a fluorescence microscope (Leica Microsystems, Oberkochen, Germany).

\section{Rat VSMC isolation and culture}

Primary rats VSMCs were isolated and cultured using previously described methods [12]. After VSMCs migrated from tissue pieces in Dulbecco's minimum essential medium (DMEM; Gibco, Grand Island, NY) supplemented with $20 \%$ fetal bovine serum (FBS; Gemini, West Sacramento, CA), they were collected and cultured in DMEM supplemented with 10\% FBS, $100 \mathrm{U} / \mathrm{mL}$ penicillin, and $100 \mathrm{mg} / \mathrm{mL}$ streptomycin. Cells were grown in a humidified incubator (Thermo Fisher Scientific) at $37^{\circ} \mathrm{C}$ in a $5 \% \mathrm{CO}_{2}$ atmosphere, and VSMCs at passages 3 to 8 were used for all experiments. VSMCs were treated with hydrogen peroxide $\left(\mathrm{H}_{2} \mathrm{O}_{2}, 0.5 \mathrm{mM}\right)$ for $12 \mathrm{~h}$ to induce oxidative damage and pre-treated with tBHQ $(20 \mu \mathrm{M} / \mathrm{mL})$ for $24 \mathrm{~h}$ and Nrf-2 inhibitor ML385 (MedChemExpress, NJ, USA) $(5 \mu \mathrm{M} / \mathrm{mL})$ for $72 \mathrm{~h}$ [13] before stimulation with $\mathrm{H}_{2} \mathrm{O}_{2}$. VSMCs were harvested for mRNA and protein analyses and functional testing. Each experiment was performed at least three times with triplicate cultures. 


\section{Cell viability and proliferation assay}

To choose appropriate concentrations of $\mathrm{H}_{2} \mathrm{O}_{2}$ and $\mathrm{tBHQ}$, cell viability was evaluated by a Cell Counting Kit-8 (CCK-8, Dojindo Molecular Technologies, Gaithersburg, MD) according to the manufacturer's instruction. Cells were seeded in 96-well plate with $5 \times 10^{3}$ cells/well and were cultured until $80 \%$ confluent. After treatment with different dosages of $\mathrm{H}_{2} \mathrm{O}_{2}(10,1,0.5,0.2,0.1 \mathrm{mM})$ for $12 \mathrm{~h}$ and $\mathrm{tBHQ}(1,10$, $20,30,40,50 \mu \mathrm{M} / \mathrm{mL}$ ) for $24 \mathrm{~h}, 10 \mu \mathrm{L}$ of CCK-8 solution was added into the culture medium of each well, and then cells were incubated in humidified $95 \%$ air and $5 \% \mathrm{CO}_{2}$ for $2 \mathrm{~h}$ at $37^{\circ} \mathrm{C}$. To evaluate the influence of $\mathrm{Nrf}-2$ signaling on proliferation, cell proliferation tests were performed as mentioned above after treatment with $\mathrm{tBHQ}$ and ML 385. Absorbance was measured at $450 \mathrm{~nm}$ using a microplate reader (Bio-Tek, Winooski, VT).

\section{Intracellular ROS measurement}

To determine intracellular ROS levels, cells were seeded in 6-well plate $\left(1 \times 10^{5}\right.$ cells/well). After treatment as described above, DCFH-DA fluorescence probes (1:1000 dilution; Beyotime, Haimen, China) were loaded, and cells were incubated for $1 \mathrm{~h}$ at $37^{\circ} \mathrm{C}$. Then, VSMCs were washed three times with serum-free DMEM and suspended in PBS. The fluorescence intensity of DCF was measured at wavelengths of $488 \mathrm{~nm}$ (excitation) and 525 $\mathrm{nm}$ (emission) in a flow cytometer (Thermo Fisher Scientific, Waltham, MA).

\section{Apoptosis analysis}

The Dead Cell Apoptosis Kit with Annexin V Alexa \& propidium iodide (PI) (Invitrogen, Carlsbad, CA) was employed to measure the apoptosis rate. Cells were treated as mentioned above, and agent-free culture was used as negative control. Next, VSMCs were harvested and washed twice with PBS. Cells were resuspended in $1 \times$ annexin-binding buffer and then stained with $5 \mu \mathrm{L}$ of PI and FITC Annexin-V for $15 \mathrm{~min}$ at room temperature in the dark. After the incubation period, $400 \mu \mathrm{L} 1 \times$ annexin-binding buffer was added, and stained cells were measured by flow cytometry, analyzing fluorescence emission at $530 \mathrm{~nm}$ and excitation at $488 \mathrm{~nm}$.

\section{Hoechst staining}

For staining, cells were seeded in 24-well plates at $2 \times$ $10^{4}$ cells/well and cultured to $60-70 \%$. After treatment, cells were fixed with fixation buffer for $10 \mathrm{~min}$. After being washed three times with PBS, cells were incubated in $0.2 \mathrm{~mL}$ Hoechst (Beyotime) for $5 \mathrm{~min}$ and then washed three times with PBS. The images were captured using a fluorescence microscope (Leica) at wavelength of 350 $\mathrm{nm}$ (excitation) and $460 \mathrm{~nm}$ (emission). The count of positive cells was analyzed by ImageJ software (National Institutes of Health, Bethesda, MD).

\section{Cell migration assay}

For wound healing assays, cells were seeded into 24-well plates, treated as described above, and cultured until $90 \%$ confluence. After scratching by a $10-\mu \mathrm{L}$ pipette tip, the wound width was photographed and measured by ImageJ at 0 and $24 \mathrm{~h}$. For transwell assays, cells suspensions of $5 \times 10^{4}$ cells $/ \mathrm{mL}$ were seeded in the upper chambers and incubated with Nrf-2 agonist or inhibitor. After treatment with $\mathrm{H}_{2} \mathrm{O}_{2}$ for $12 \mathrm{~h}$, DMEM with $10 \%$ FBS was added to the bottom chambers to stimulate migration. Cells in the upper chambers were scraped after $24 \mathrm{~h}$. Migrated cells were fixed with 4\% PFA and stained in crystal violet. Numbers of migrated cells were assessed under light microscopy by counting six fields.

\section{RNA isolation and quantitative real-time polymerase chain reaction (qRT-PCR) analysis}

Total RNA was extracted by using TRIzol (Takara, Kusatsu, Japan) according to the manufacturer's protocol. For qRT-PCR analysis, cDNA was synthesized using ABScript II cDNA First-Strand Synthesis Kit under to manufacturer instructions. mRNA expression was evaluated with SYBR green-based PCR reactions. The results were analyzed using the $\Delta \Delta \mathrm{Ct}$ method and expressed as ratio of the internal control, glyceraldehyde 3-phosphate dehydrogenase (GAPDH).

\section{Cytokine and chemokine analysis}

Cellular supernatants were collected (centrifuge at $1000 \mathrm{~g}$ for $5 \mathrm{~min}$ ) after cells were treated as mentioned above. We choose interleukin 1 beta (IL-1b), IL-6, monocyte chemoattractant protein 1 (MCP-1), and tumor necrosis factor alpha (TNF-a). Cytokines were measured via a MILLIPLEX MAP Rat CVD Panel (Millipore, Billerica, MA).

\section{Western blot analysis}

The proteins were collected using RIPA lysis buffer. To determine the expression of nuclear transcription factor Nrf-2, nuclear and cytoplasmic proteins were extracted separately. Cell lysates were separated by sodium dodecyl sulfate-polyacrylamide gel electrophoresis and then transferred to polyvinylidene fluoride membranes. After membranes were blocked, proteins were detected with the following primary antibodies (Abcam, Cambridge, $\mathrm{UK}$ ): anti-Nrf-2 (Ab137550), anti-NAD(P)H quinone dehydrogenase 1 (NQO-1) (ab28947), anti-SM22 $\alpha$ (ab14106), and anti- $\alpha$ SMA (ab7817). The membranes were exposed to X-ray after being probed with enhanced chemiluminescence and visualized with an imaging system (Tanon, Shanghai, China). The results were calculated by ImageJ software. 


\section{Statistical analysis}

Each experiment was performed at least three times with triplicate groups. Data are the means \pm standard errors of the mean. All statistical analyses were performed using one-way analysis of variance in IBM SPSS statistics 23 (IBM Corp., Armonk, NY), and figures were generated in GraphPad Prism software version 7.0 (GraphPad Inc., San Diego, CA). $p<0.05$ was considered significant.

\section{Results}

Nrf-2 signaling can inhibit IA formation and progression in a rat IA model

We first assessed the effect of Nrf-2 activation on IA formation and progression in a rat model. Figure $1 \mathrm{~B}$ (a, b) HE staining show vessel wall weakness. Fig 1B shows the degradation of vessel wall and myointimal hyperplasia which are characteristics of unruptured intracranial aneurysm wall. Compared with normal cerebral artery, thinning of tunica media is more significant in the experimental group. Figure 1A (a) through (e) show normal cerebral arteries, aneurysmal arteries, an aneurysm, multiple aneurysms, and a ruptured aneurysm, respectively. The aneurysms are more likely to occur at major branching brain arteries. We found the rat aneurysms located at bifurcation of Willis' circle and anterior circulation. There were seven and one aneurysms formed in the control (one middle cerebral artery, five anterior circulation, and one posterior circulation) and tBHQ (one anterior circulation) groups, respectively. These five types of arteries corresponded to different scores. The aneurysmal score of the tBHQ group was much lower than in the control group, suggesting that $\mathrm{tBHQ}$ can decrease the incidence of aneurysm and rate of rupture. There was a significant difference in aneurysm incidence between the control and tBHQ groups. The Nrf-2 agonist tBHQ significantly inhibited IA formation and progression in vivo.

\section{Downregulation and nuclear translocation of Nrf-2 in human IA specimens}

DAB staining demonstrated decreased Nrf-2 expression in IAs compared with STAs. Furthermore, Nrf-2 expression overlapped with the nucleus position in IA, indicating that more Nrf-2 translocated into the nucleus in IAs than STAs (Fig. 2A).
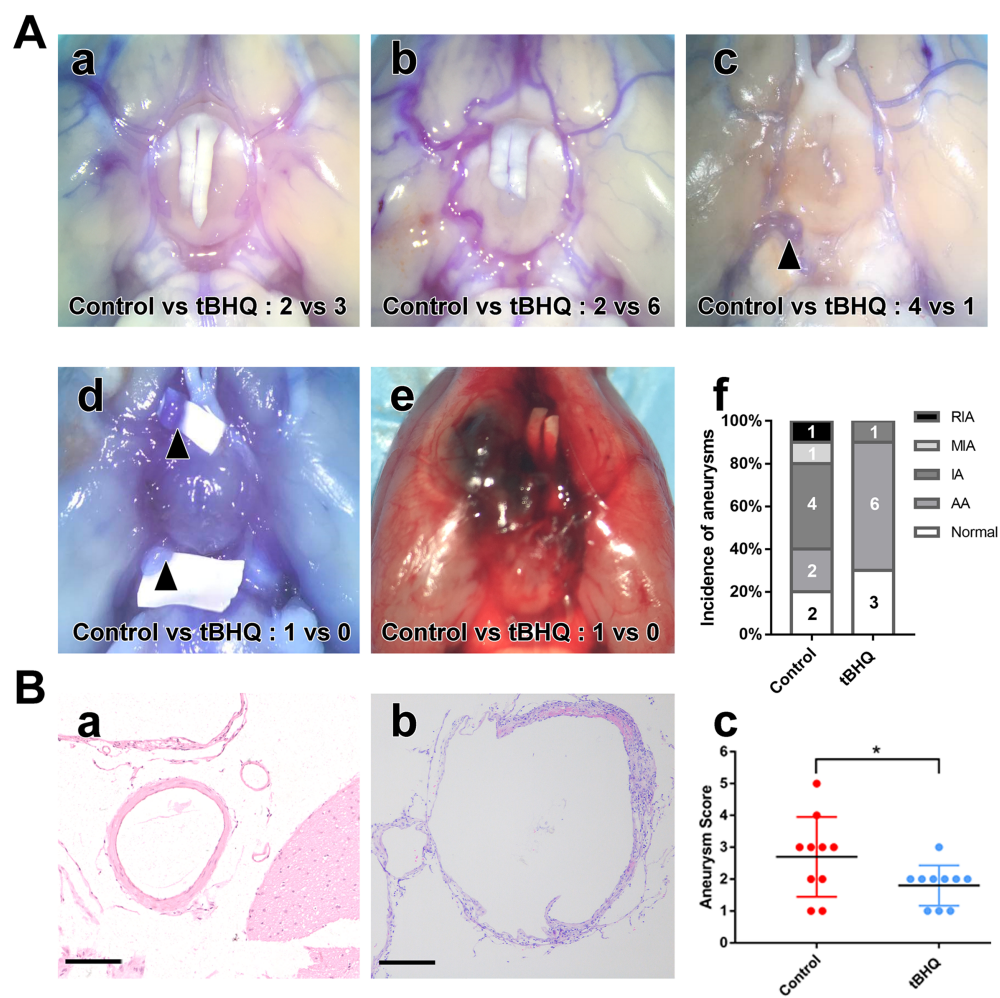

Fig. 1 The effect of Nrf-2 activation on IA formation and progression. a Rate of aneurysm formation and rupture in the control and tBHQ treatment groups. (a) normal vessels, (b) aneurysmal artery (AA), (c) aneurysm (IA), (d) multiple aneurysms (MIA), (e) ruptured aneurysm (MIA), (f) quantitative analysis of (a) through (e) in the control and tBHQ treatment groups ( $N=$ intracranial aneurysms amounts under different condition). b (a) HE staining of normal cerebral artery $(\mathrm{bar}=50 \mu \mathrm{M}$ ), (b) HE staining of IA (scale bar $=50 \mu \mathrm{M}$ ), (c) aneurysmal scores of the control and tBHQ treatment groups. Aneurysmal scores in the control group were much higher than that in the tBHQ group. (a) through (e). A corresponding to aneurysmal score of 1 to $5 . * 0<0.05$ compared with the control group 
VSMCs switched to the synthetic phenotype in human IA tissues

Based on previous studies, we chose SM22 $\alpha$ and $\alpha$ SMA as markers of contractile phenotype VSMCs and found that levels of both were decreased in the synthetic phenotype. In STA, high immunofluorescent signals of SM22 $\alpha / \alpha$ SMA were detected in VSMCs with normal cellular morphology (Fig. 2B). However, SM22 $\alpha$ and $\alpha$ SMA signals obviously declined in VSMCs in aneurysm walls (Fig. 2B), suggesting that VSMCs in IA walls switched to the synthetic phenotype.

\section{Nrf-2 agonist tBHQ can help VSMCs maintain a contractile phenotype}

As mentioned above, the Nrf-2 activator tBHQ can reduce IA formation and inhibit progression in a rat IA model. SM22 $\alpha$ and $\alpha$ SMA expression in aneurysms we developed in the control and tBHQ groups were

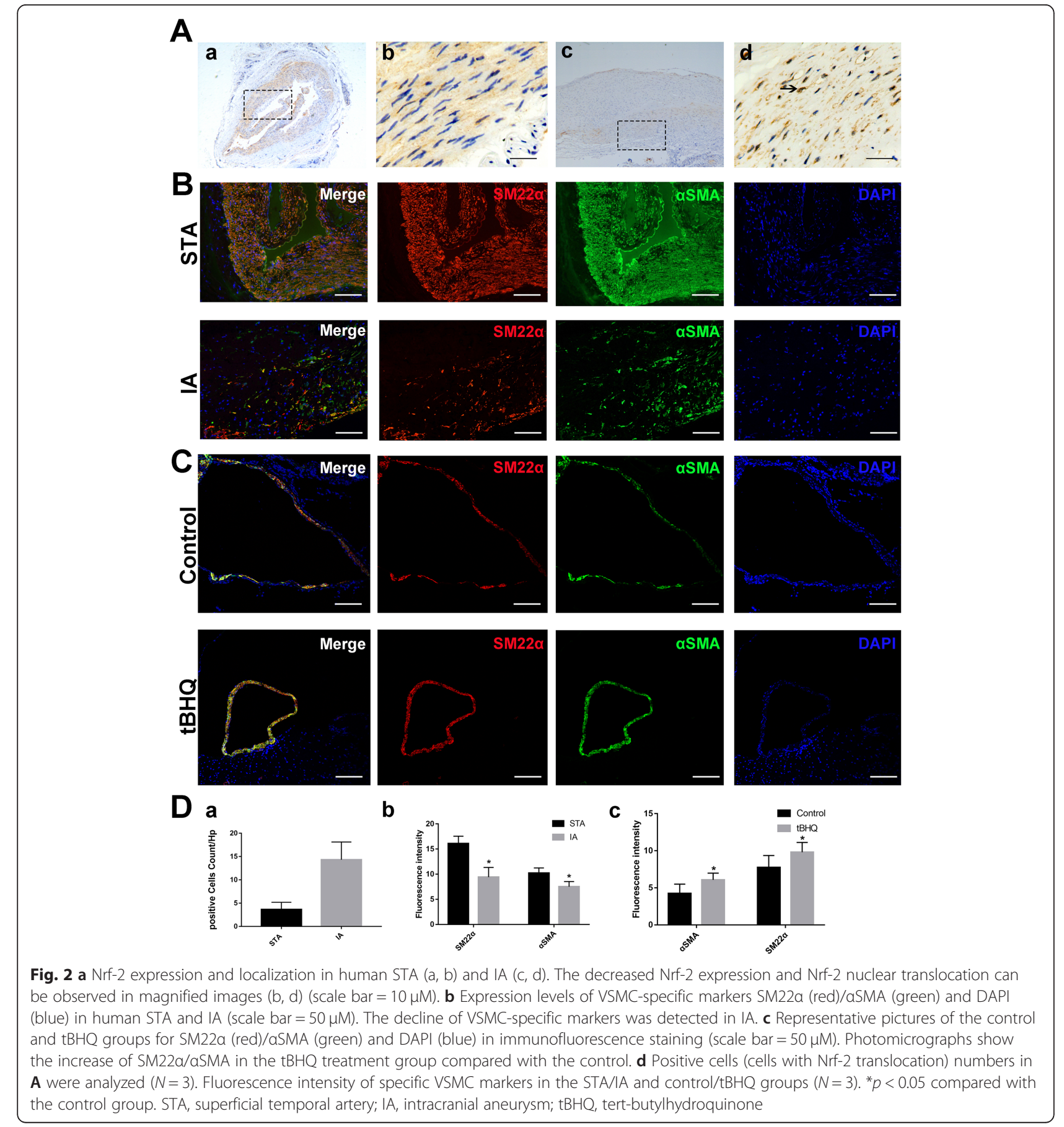


analyzed. Aneurysms in the tBHQ group were smaller in diameter and had a thicker wall, a higher proportion of VSMCs, and greater SM22 $\alpha / \alpha$ SMA expression (Fig. 2C). The results indicated that $\mathrm{tBHQ}$ prevented VSMCs from decreasing in number and changing to the synthetic phenotype.

\section{$\mathrm{H}_{2} \mathrm{O}_{2}$ caused oxidative damage in VSMCs}

We initially intended to develop a VSMC oxidative damage model by incubation with $\mathrm{H}_{2} \mathrm{O}_{2}$ in vitro. $\mathrm{ROS}$ upregulation is known to cause oxidative damage. Cell viability was detected by CCK- 8 assays, and intracellular ROS levels were by measured by flow cytometry. As shown in Fig. 3A (a) and Fig. 3B, $\mathrm{H}_{2} \mathrm{O}_{2}(0.5 \mathrm{mM})$ induced obvious intracellular ROS accumulation but had little influence on cell viability $(p<0.05)$. This concentration was therefore used in subsequent experiments.

Nrf-2 activator tBHQ and Nrf-2 inhibitor ML 385 decreased and increased cellular ROS levels caused by $\mathrm{H}_{2} \mathrm{O}_{2}$, respectively

To access the protective effect of tBHQ on $\mathrm{H}_{2} \mathrm{O}_{2}$-induced cytotoxicity, VSMCs were treated with different $\mathrm{tBHQ}$ concentrations. At $20 \mu \mathrm{M} / \mathrm{mL}$, tBHQ did not affect VSMC survival rate (Fig. 3A (b), $p<0.05$ ). ML 385 treatment was performed as described by Biswal et al. [13]. Figure 3C shows $\mathrm{H}_{2} \mathrm{O}_{2}(0.5 \mathrm{mM})$ increased ROS levels yielding a twofold higher fluorescence intensity compared with the control group, but this was significantly reduced by $\mathrm{tBHQ}(p<0.05)$. In contrast, intracellular ROS in the ML 385 group increased threefold compared to the $\mathrm{H}_{2} \mathrm{O}_{2}$ treatment group $(p<0.05)$. These results suggest that activation or inhibition of Nrf-2 could affect intracellular oxidative status.

\section{Nrf-2 inhibition contributed to VSMC proliferation and migration}

Proliferation and migration abilities are different in the two distinct smooth muscle cell phenotypes [14]. Generally, synthetic VSMCs exhibit higher growth and migratory activity than contractile VSMCs [15]. We assessed cell viability after $12 \mathrm{~h}$ of $\mathrm{H}_{2} \mathrm{O}_{2}$ treatment with or without Nrf-2 activation. We found VSMCs oxidized by $\mathrm{H}_{2} \mathrm{O}_{2}$ exhibited higher proliferation ability, while tBHQ attenuated the $\mathrm{H}_{2} \mathrm{O}_{2}$-induced growth rate increase. Conversely, ML 385 pretreatment exacerbated this effect (Fig. 4A, $p<0.05$ ). VSMC motility was analyzed by wound healing and transwell assays. The results (Fig. 4B and C) demonstrated that oxidative damage could enhance VSMC migration, and ML 385 treatment promoted

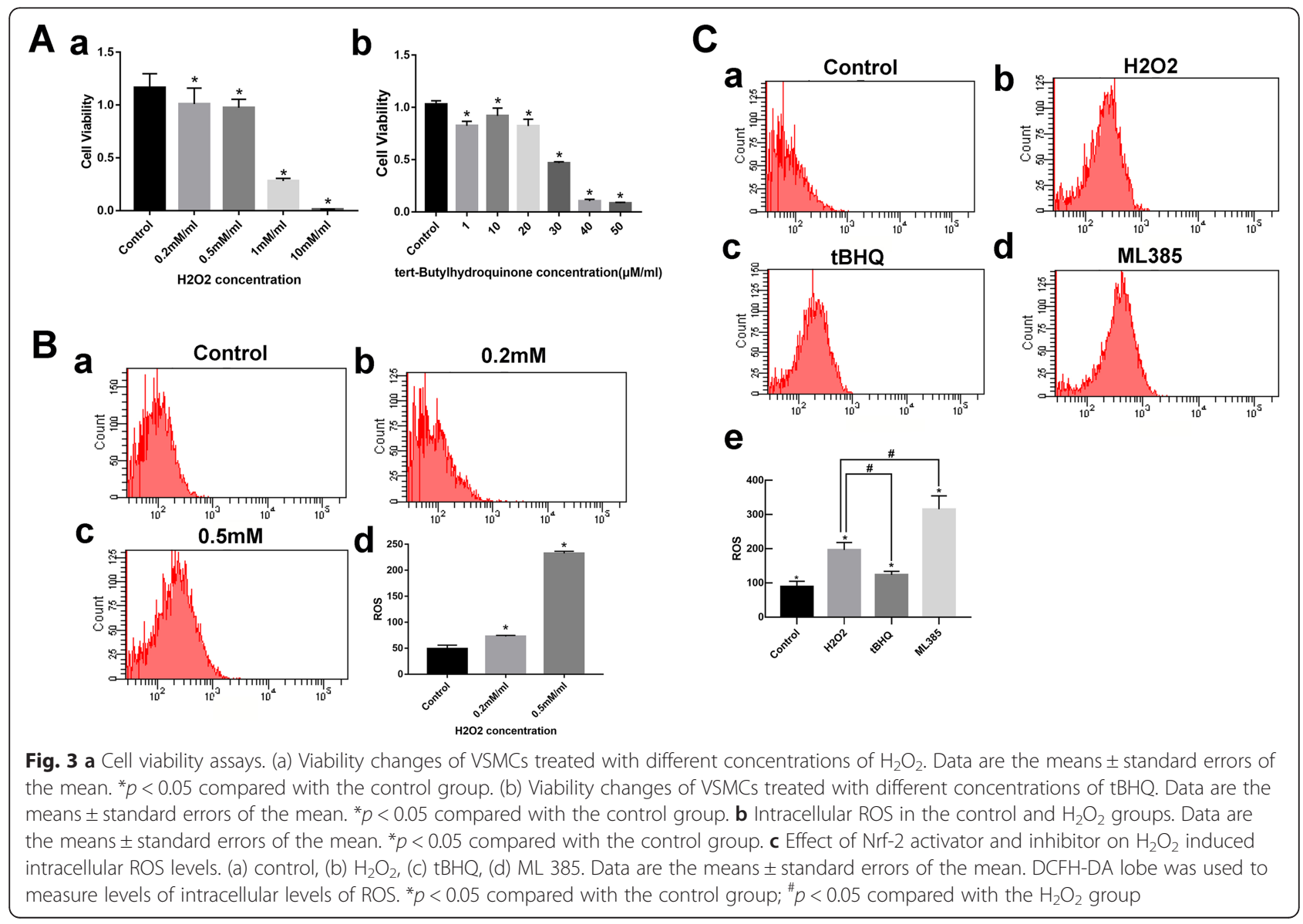




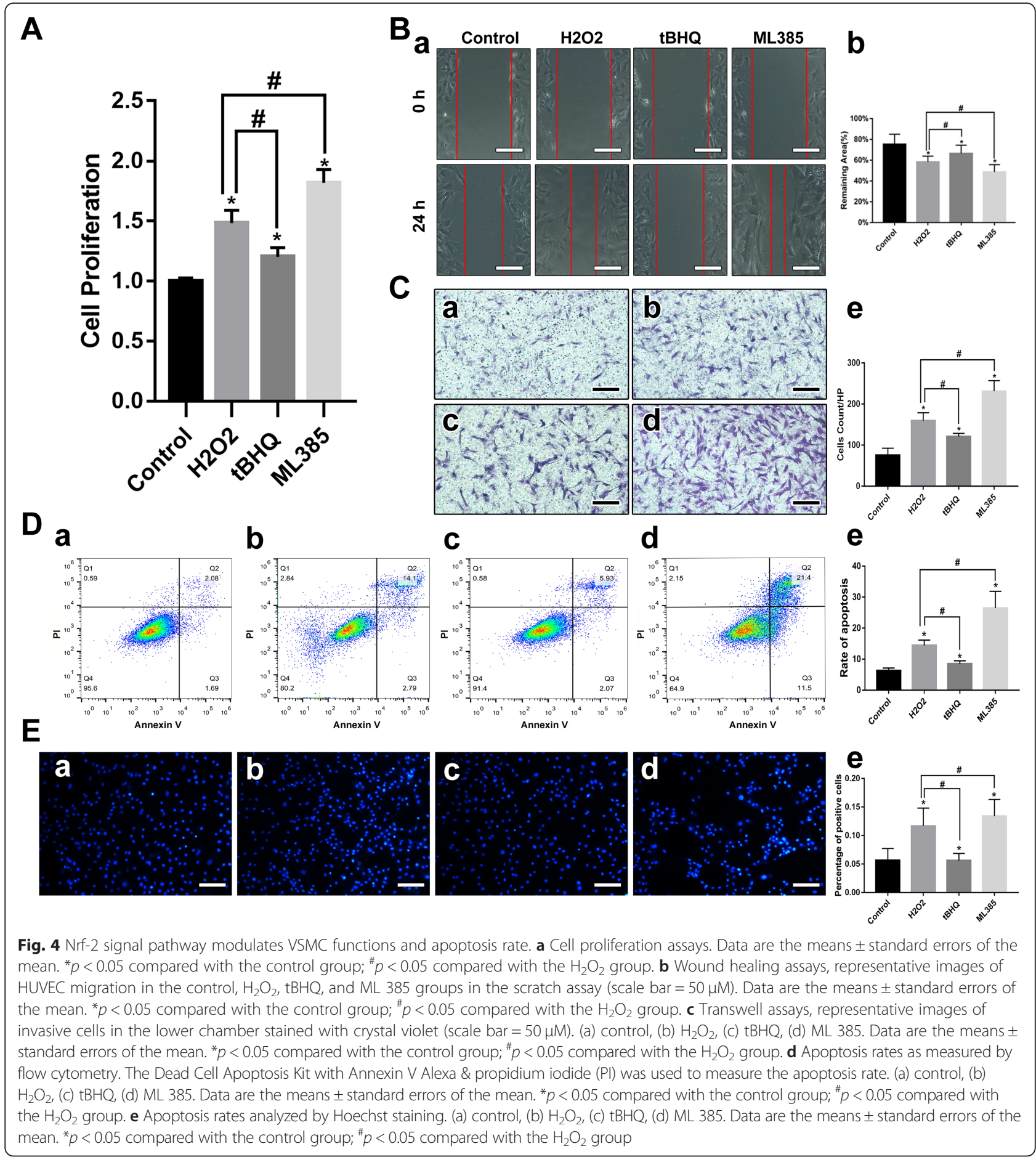

migratory activity compared with the $\mathrm{H}_{2} \mathrm{O}_{2}$ group. A significant inhibition of migration was observed when VSMCs were incubated with tBHQ $(p<0.05)$. These results indicate that Nrf-2 could modulate VSMC proliferation and migration by regulating cellular oxidative stress.
Effect of tBHQ and ML 385 on $\mathrm{H}_{2} \mathrm{O}_{2}$-induced vascular smooth muscle cells apoptosis

Oxidative stress leads to apoptosis. Hoechst staining and flow cytometry were performed to examine the apoptosis rate. As predicted, the VSMC apoptosis rate increased significantly after treatment with $\mathrm{H}_{2} \mathrm{O}_{2}$. 
Next, we detected the effect of Nrf-2 signaling on $\mathrm{H}_{2} \mathrm{O}_{2}$-induced VSMC apoptosis. Consistent with the effect of Nrf-2 on oxidative stress, tBHQ suppressed apoptosis, while ML 385 promoted apoptosis by inhibiting Nrf-2 (Fig. 4D and E, $p<0.05$ ).

Nrf-2 activation improves VSMC-specific gene expression Several studies have described how oxidative stress can induce VSMCs to switch from the contractile to synthetic type. However, the role of Nrf-2, which is one of the most important antioxidant molecules in phenotypic modulation, is still unclear. Thus, qRT-PCR and western blot were performed to investigate the effect of Nrf- 2 on the expression of VSMC-specific markers. To investigate whether tBHQ activates Nrf-2 or ML 385 inhibits Nrf-2, we analyzed Nrf-2 levels in the cytoplasm and nucleus. As shown in Fig. 5D, cytoplasmic Nrf-2 decreased and nuclear Nrf-2 increased after tBHQ treatment $(p<0.05)$. Conversely, ML 385 inhibited Nrf-2 nuclear translocation $(p<0.05)$. SM $22 \alpha$ and $\alpha$ SMA mRNA and protein levels decreased in $\mathrm{H}_{2} \mathrm{O}_{2}$-incubated VSMCs. The inhibition of SM22 $\alpha$ and $\alpha$ SMA was largely diminished in VSMCs pretreated with $\mathrm{tBHQ}$, which was consistent with $\mathrm{tBHQ}$-induced Nrf-2 nuclear translocation, whereas the Nrf-2 inhibitor ML 385 further exacerbated the decrease in both markers (Fig. 5A (a-c), Fig. 5C, $p<0.05$ ).

\section{Impact of Nrf-2 activation on oxidative redox balance and inflammation}

Nrf-2 is an important endogenous antioxidant defense molecule. Many enzymes downstream of the Nrf- 2 pathway are primary components of the antioxidant system, also known as phase II detoxifying enzymes. The antioxidant enzymes examined by qRT-PCR and western blot included NQO-1, glutamate-cysteine ligase catalytic subunit (GCLC), and superoxide dismutase 1 (SOD 1). Inflammatory cytokines and oxidative stress are closely related. qRT-PCR and MILLIPLEX MAP were performed to detect inflammatory cytokines including IL1b, IL-6, MCP-1, and TNF-a. Figure 5A and B shows that $\mathrm{tBHQ}$ increased antioxidant enzyme gene expression and downregulated cytokine levels by activating Nrf-2 $(p<0.05)$. ML 385 aggravated oxidative damage by upregulating inflammatory cytokines and inhibited the transcription of genes encoding antioxidant enzymes $(p<0.05)$.

\section{Discussion}

The mechanism of IA development is still unclear. Many pathological factors like oxidative stress, inflammation, dysregulated hemodynamics, and extracellular matrix degeneration contribute to their formation and progression [16]. The interaction between oxidative stress and inflammation is a key mechanism of endothelial dysfunction and arterial damage. A role of VSMCs phenotype switching has been described in IAs, atherosclerosis, and aortic aneurysms. Oxidative stress can accelerate inflammation directly and indirectly, which further increases oxidative stress. This can induce phenotypically switched VSMCs to stimulate pro-inflammatory processes, extracellular matrix remodeling, and mural cells apoptosis, all of which eventually contribute to IA formation and rupture. Numerous lines of evidence indicate that VSMC phenotypic modulation is associated with IA formation, growth, and rupture [17-19].

Nrf-2, a basic leucine zipper transcription factor, plays a critical role in modulating cellular redox balance by promoting the transcription of detoxifying and antioxidant genes. Under oxidative conditions, Nrf-2 separates from Keap1 in the cytoplasm and translocates to the nucleus, which leads to the transcription of phase II detoxification enzymes and anti-inflammatory proteins. Researchers previously demonstrated that VSMCs phenotypes are influenced by inhibiting ROS generation, and this is important for maintaining vascular homeostasis [20]. Other findings also suggest that Nrf-2 could affect VSMC functions by reducing oxidative stress [21, 22]. We hypothesized that Nrf-2 pathway activation can prevent VSMCs from switching to the synthetic type by modulating oxidative stress and exerting protective effects against IA development.

We first examined the role of Nrf-2 pathway activation in vivo. An elastase-induced rat IA model was developed, and arteries were divided into five different types: normal cerebral arteries, aneurysmal arteries, aneurysms, multiple aneurysms, and ruptured aneurysms. After 30 days of treatment with $\mathrm{Nrf}-2$ agonist $\mathrm{tBHQ}$, there were lower aneurysmal scores and less IA formation and rupture compared with the control (Fig. 1). This indicates that Nrf-2 activation can suppress IA formation and progression. Consistent with our data, Zeng et al. reported that increased Nrf-2 levels help inhibit acute aortic dissection formation, which involves a pathological mechanism similar to IA [23]. In the presence of stimulating factors, Nrf-2 translocates into the nucleus and promotes downstream target genes expression by binding to the antioxidant response element. Our immunohistochemical results revealed stabilized Nrf-2 distributed in the cytoplasm of STAs, while downregulation and nuclear translocation were found in aneurysm walls (Fig. 2A). Nakajima et al. detected VSMCs phenotypic modulation in human aneurysmal walls and concluded that this process was related to aneurysm remodeling and rupture [24]. We found VSMC morphological changes, decreased SM22 $\alpha / \alpha S M A$ expression, and loss of mural VSMCs in IA, all of which are characteristic of IA walls (Fig. 2B). In the rat IA model, SM22 $\alpha / \alpha$ SMA was upregulated and aneurysm diameter was smaller 


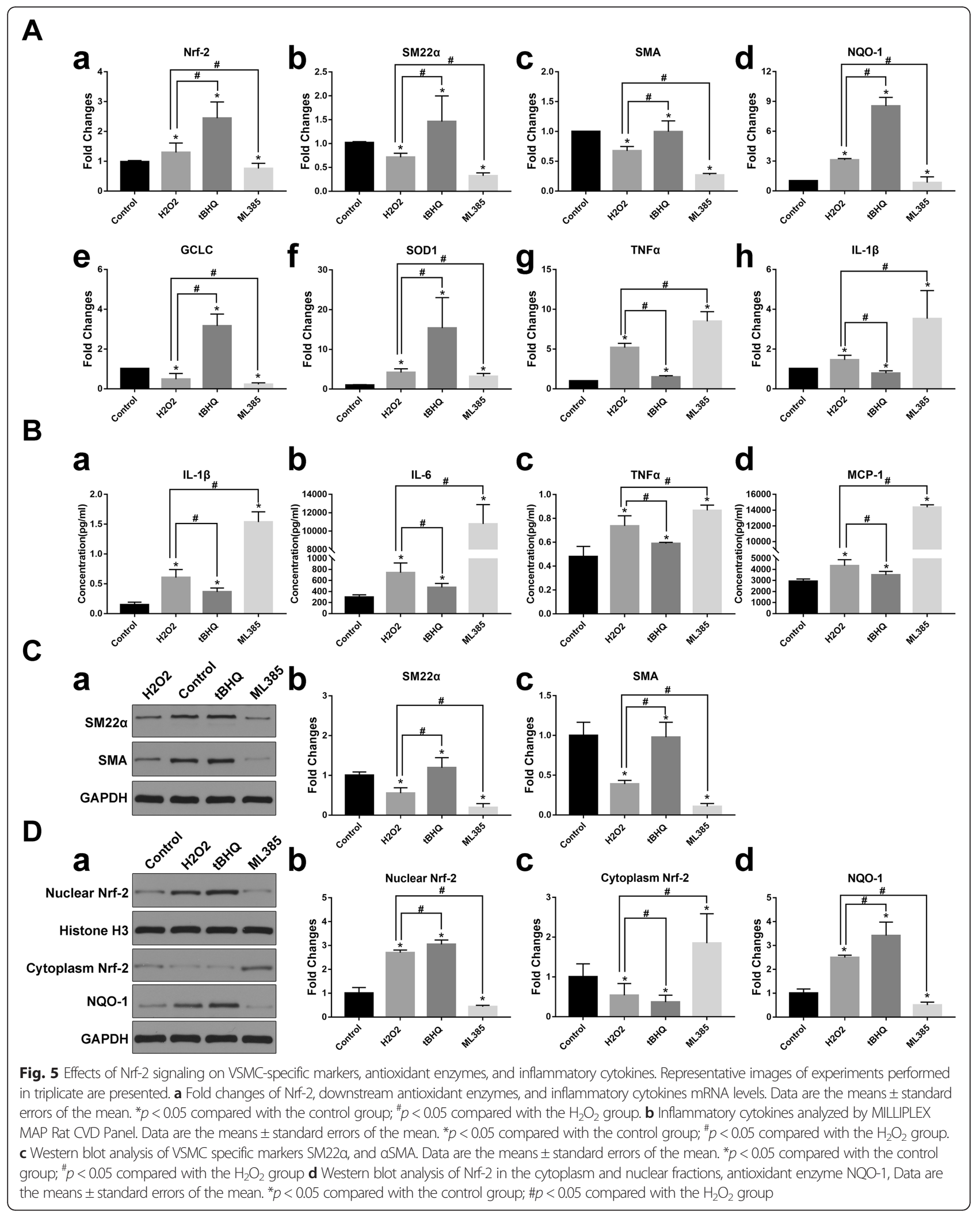




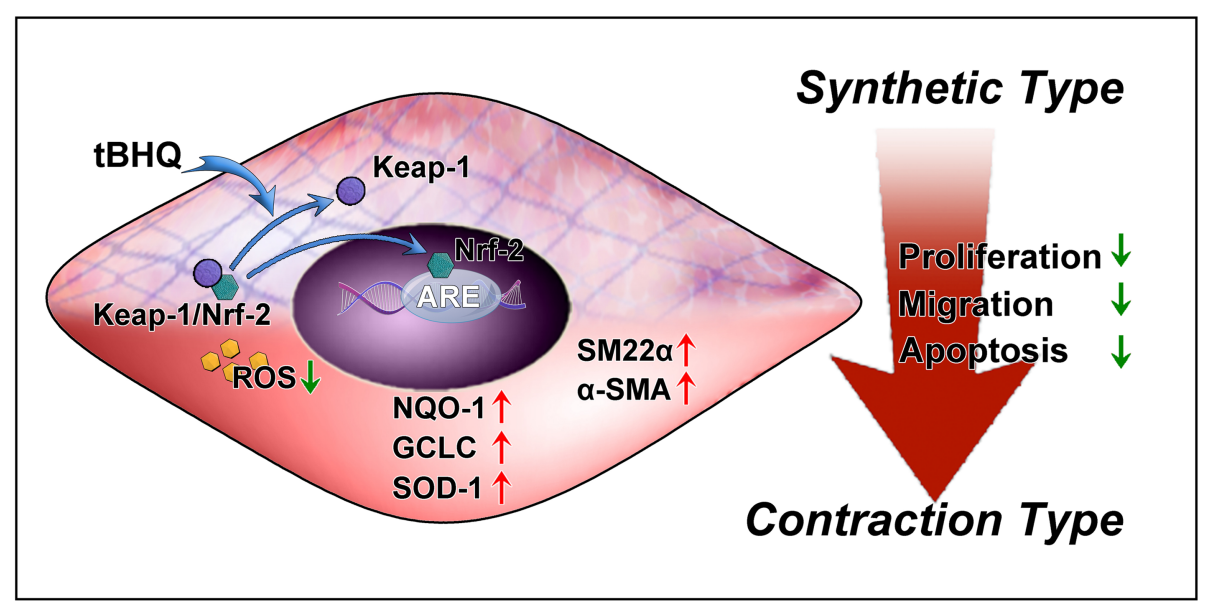

Fig. 6 Schematic illustrating how Nrf-2 activation regulates phenotypic modulation. Under oxidative-stimulating conditions, Nrf-2 is activated and translocates into the nucleus. Nrf-2 signal pathway activation promotes expression of VSMC-specific markers SM22a and aSMA. The underlying mechanism may be that Nrf-2 upregulates expression levels of its downstream antioxidant enzymes and inhibits pro-inflammatory cytokines

after tBHQ treatment (Fig. 2C). These results suggest that Nrf-2 activation inhibits IA development by modulating VSMCs phenotype.

To investigate how Nrf-2 contributes to VSMC phenotypic switching, we examined the effect of Nrf-2 activation or inhibition on VSMC phenotype changes and functions in vitro. $\mathrm{H}_{2} \mathrm{O}_{2}$ can promote ROS generation, which is a central molecular component of oxidative stress and is widely used to induce cellular damage $[25,26]$. We selected tBHQ as a Nrf2 activator and ML 385 as Nrf-2 inhibitor, since they are well-studied and specific for Nrf-2 [13, 27, 28]. We observed that $\mathrm{tBHQ}$ decreased ROS generation while ML 385 promoted significant ROS accumulation (Fig. 3C). The results of cell proliferation and migration tests revealed that Nrf-2 activation exerts a negative effect on proliferative and migratory abilities. Two different methods confirmed that Nrf-2 activation decreased apoptosis (Fig. 4). Therefore, Nrf-2, which has regulatory roles in VSMC proliferation, migration, and apoptosis, is also associated with vascular remodeling that leads to IA formation and progression. We demonstrated that VSMCs switched to the synthetic phenotype after oxidative treatment, but while tBHQ helped, VSMCs maintain contractile phenotype according to qRT-PCR and western blot results. Conversely, ML 385 profoundly suppressed VSMCspecific marker expression (Fig. 5). The underlying mechanism may involve upregulation of antioxidant enzymes, decreased pro-inflammatory cytokine expression, and less intracellular ROS accumulation (Fig. 3, Fig. 5). When SM22 $\alpha / \alpha S M A$ levels are decreased, VSMCs develop increased capacities for proliferation, migration, and secretion of pro-remodeling factors and pro-inflammatory cytokines $[29,30]$. VSMCs undergoing phenotypic switching can also aggravate oxidative stress and inflammation. These results are consistent with the functional changes described above and suggest that Nrf-2 modulates VSMC phenotype switches by affecting antioxidant and anti-inflammatory protein expression.

\section{Conclusion}

In summary, our results provide novel evidence that Nrf-2 signaling can inhibit IA formation and progression by regulating VSMC phenotypes. The Nrf-2 agonist tBHQ decreases the rates of IA formation and rupture and increases VSMC contractile gene expression by promoting Nrf-2 nuclear translocation (Fig. 6). The Nrf-2 antagonist ML 385 reverses this effect. Although prior studies have found a close relationship between VSMC phenotype switching and IA formation and rupture, none have assessed the role of the Nrf-2 signal pathway. Our findings provide a theoretical basis for research into novel pharmacologic therapies.

\section{Abbreviations}

ECM: Extracellular matrix; HE: Hematoxylin and eosin; IA: Intracranial aneurysm; MHC: Myosin heavy chain; NQO-1: NAD(P)H quinone dehydrogenase 1; Nrf-2: Nuclear factor erythroid 2-related factor 2; PCR: Polymerase chain reaction; ROS: Reactive oxygen species; SM22a: Smooth muscle 22 alpha; SM-a-actin: Smooth muscle alpha actin; tBHQ: Tert-butylhydroquinone; VSMC: Vascular smooth muscle cell

\section{Acknowledgements}

Not applicable.

\section{Authors' contributions}

YS contributed to the conception and design of study, collection and/or assembly of data, data analysis and interpretation, and manuscript writing. SL contributed to the database input and data interpretation, manuscript writing, and final approval of manuscript. YS contributed to the data analysis and interpretation and manuscript writing. PL contributed to the data collection, manuscript writing, and analysis. $Z Y$ and $Y L$ contributed to the animal experiments and data collection. KQ, GY, and ZF contributed to the in vitro assay data collection and analysis and histological examination. WZ contributed to the conception and design of study and revision and final approval of manuscript. WZ is the corresponding author of this article. All authors read and approved the final manuscript. 


\section{Funding}

This study was supported by The Outstanding Academic Leaders Program of Shanghai Municipal Commission of Health and Family Planning (No.2017BR006), Shanghai Sailing Program Project (No.16YF1401200), and National Natural Science Foundation of China (No.81801148, No.81870911, and No.81571102)

\section{Availability of data and materials}

Data generated and analyzed as part of this study are included in the manuscript or are available upon request from the corresponding author.

\section{Ethics approval and consent to participate}

All animal procedures were carried out according to the protocol of our Institutional Animal Care and Use Committee. The experimental protocol was reviewed and approved by the Ethics Committee of the Huashan Hospital affiliated with Fudan University in Shanghai, People's Republic of China.

\section{Consent for publication}

Not applicable.

\section{Competing interests}

The authors declare that they have no competing interests.

\section{Author details}

${ }^{1}$ Department of Neurosurgery, Huashan Hospital, Fudan University, 12 Wulumiqi Rd., Shanghai 200040, People's Republic of China. ${ }^{2}$ Department of Neurology, Ruijin Hospital, Shanghai Jiao Tong University School of Medicine, 197 Ruijin Rd. No.2, Shanghai 200025, China.

\section{Received: 10 June 2019 Accepted: 29 August 2019}

Published online: 04 October 2019

\section{References}

1. Etminan N, Rinkel GJ. Unruptured intracranial aneurysms: development, rupture and preventive management. Nat Rev Neurol. 2016;12(12):699-713. https://doi.org/10.1038/nrneurol.2016.150.

2. Chalouhi N, Hoh BL, Hasan D. Review of cerebral aneurysm formation growth, and rupture. Stroke. 2013;44(12):3613-22. https://doi.org/10.1161/ STROKEAHA.113.002390.

3. Signorelli F, Sela S, Gesualdo L, et al. Hemodynamic stress, inflammation, and intracranial aneurysm development and rupture: a systematic review. World Neurosurg. 2018;115:234-44. https://doi.org/10. 1016/j.wneu.2018.04.143

4. Liu P, Song Y, Zhou Y, et al. Cyclic mechanical stretch induced smooth muscle cell changes in cerebral aneurysm progress by reducing collagen type IV and collagen type VI levels. Cell Physiol Biochem. 2018;45(3):105160. https://doi.org/10.1159/000487347.

5. Starke RM, Chalouhi N, Ding D, et al. Vascular smooth muscle cells in cerebral aneurysm pathogenesis. Transl Stroke Res. 2014;5(3):338-46. https:// doi.org/10.1007/s12975-013-0290-1.

6. Wang $G$, Jacquet $L$, Karamariti $E, X u$ Q. Origin and differentiation of vascular smooth muscle cells. J Physiol. 2015;593(14):3013-30. https://doi.org/10. 1113/JP270033.

7. Wall VZ, Bornfeldt KE. Arterial smooth muscle. Arterioscler Thromb Vasc Biol. 2014;34(10):2175-9. https://doi.org/10.1161/ATVBAHA.114.304441.

8. Starke RM, Chalouhi N, Ali MS, et al. The role of oxidative stress in cerebral aneurysm formation and rupture. Curr Neurovasc Res. 2013:10(3):247-55.

9. Galougahi KK, Ashley EA, Ali ZA. Redox regulation of vascular remodeling. Cell Mol Life Sci. 2016;73(2):349-63. https://doi.org/10.1007/s00018-0152068-y.

10. Keleku-Lukwete N, Suzuki M, Yamamoto M. An overview of the advantages of KEAP1-NRF2 system activation during inflammatory disease treatment. Antioxid Redox Signal. 2018;29(17):1746-55. https:// doi.org/10.1089/ars.2017.7358.

11. Nuki Y, Tsou T-L, Kurihara C, Kanematsu M, Kanematsu Y, Hashimoto T. Elastase-induced intracranial aneurysms in hypertensive mice. Hypertens. 2009;54(6):1337-44. https://doi.org/10.1161/HYPERTENSIONAHA.109.138297.

12. Zhu Q, Ni X-Q, Lu W-W, et al. Intermedin reduces neointima formation by regulating vascular smooth muscle cell phenotype via CAMP/PKA pathway.
Atherosclerosis. 2017;266:212-22. https://doi.org/10.1016/j.atherosclerosis. 2017.10.011.

13. Singh A, Venkannagari S, Oh KH, et al. Small molecule inhibitor of NRF2 selectively intervenes therapeutic resistance in KEAP1-deficient NSCLC tumors. ACS Chem Biol. 2016;11(11):3214-25. https://doi.org/10.1021/ acschembio.6b00651.

14. Davis-Dusenbery BN, Wu C, Hata A. Micromanaging vascular smooth muscle cell differentiation and phenotypic modulation. Arterioscler Thromb Vasc Biol. 2011;31(11):2370-7. https://doi.org/10.1161/ATVBAHA.111.226670.

15. Rensen SSM, Doevendans PAFM, van Eys GJJM. Regulation and characteristics of vascular smooth muscle cell phenotypic diversity. Neth Heart J. 2007;15(3):100-8.

16. Weir B. Unruptured aneurysms. J Neurosurg. 2002;97(5):1011-3. https://doi. org/10.3171/jns.2002.97.5.1011.

17. Sun L, Zhao M, Zhang J, et al. MiR-29b downregulation induces phenotypic modulation of vascular smooth muscle cells: implication for intracranial aneurysm formation and progression to rupture. Cell Physiol Biochem. 2017; 41(2):510-8. https://doi.org/10.1159/000456887.

18. Frosen J, Piippo A, Paetau A, et al. Remodeling of saccular cerebral artery aneurysm wall is associated with rupture: histological analysis of 24 unruptured and 42 ruptured cases. Stroke. 2004;35(10):2287-93. https://doi. org/10.1161/01.STR.0000140636.30204.da.

19. Chalouhi N, Ali MS, Jabbour PM, et al. Biology of intracranial aneurysms: role of inflammation. J Cereb Blood Flow Metab. 2012;32(9):1659-76. https://doi. org/10.1038/jcbfm.2012.84

20. Zhong L, He X, Si X, et al. SM22alpha (smooth muscle 22alpha) prevents aortic aneurysm formation by inhibiting smooth muscle cell phenotypic switching through suppressing reactive oxygen species/NF-kappaB (nuclear factor-kappaB). Arterioscler Thromb Vasc Biol. 2019;39(1):e10-25. https://doi. org/10.1161/ATVBAHA.118.311917.

21. Levonen $A-L$, Inkala $M$, Heikura $T$, et al. Nrf2 gene transfer induces antioxidant enzymes and suppresses smooth muscle cell growth in vitro and reduces oxidative stress in rabbit aorta in vivo. Arterioscler Thromb Vasc Biol. 2007;27(4):741-7. https://doi.org/10.1161/01.ATV.0000258868.80079.4d.

22. Ashino T, Yamamoto M, Yoshida T, Numazawa S. Redox-sensitive transcription factor Nrf2 regulates vascular smooth muscle cell migration and neointimal hyperplasia. Arterioscler Thromb Vasc Biol. 2013;33(4):760-8. https://doi.org/10.1161/ATVBAHA.112.300614.

23. Liu W, Wang B, Wang T, et al. Ursodeoxycholic acid attenuates acute aortic dissection formation in angiotensin II-infused apolipoprotein E-deficient mice associated with reduced ROS and increased Nrf2 levels. Cell Physiol Biochem. 2016;38(4):1391-405. https://doi.org/10.1159/000443082.

24. Nakajima N, Nagahiro S, Sano T, Satomi J, Satoh K. Phenotypic modulation of smooth muscle cells in human cerebral aneurysmal walls. Acta Neuropathol. 2000;100(5):475-80.

25. Arcanjo NO, Andrade MJ, Padilla P, Rodriguez A, Madruga MS, Estevez M. Resveratrol protects lactobacillus reuteri against $\mathrm{H} 2 \mathrm{O} 2$ - induced oxidative stress and stimulates antioxidant defenses through upregulation of the dhaT gene. Free Radic Biol Med. 2019;135:38-45. https://doi.org/10.1016/j. freeradbiomed.2019.02.023.

26. Lee J, Song K, Huh E, Oh MS, Kim YS. Neuroprotection against 6-OHDA toxicity in PC12 cells and mice through the Nrf2 pathway by a sesquiterpenoid from Tussilago farfara. Redox Biol. 2018;18:6-15. https://doi. org/10.1016/j.redox.2018.05.015

27. Zagorski JW, Turley AE, Freeborn RA, et al. Differential effects of the Nrf2 activators $\mathrm{tBHQ}$ and CDDO-Im on the early events of T cell activation. Biochem Pharmacol. 2018:147:67-76. https://doi.org/10.1016/j.bcp.2017.11.005.

28. Li S, Li J, Shen C, et al. Tert-Butylhydroquinone (tBHQ) protects hepatocytes against lipotoxicity via inducing autophagy independently of Nrf2 activation. Biochim Biophys Acta. 2014;1841(1):22-33. https://doi.org/10. 1016/j.bbalip.2013.09.004

29. Alexander MR, Owens GK. Epigenetic control of smooth muscle cell differentiation and phenotypic switching in vascular development and disease. Annu Rev Physiol. 2012;74:13-40. https://doi.org/10.1146/annurev-physiol-012110-142315.

30. Bennett MR, Sinha S, Owens GK. Vascular smooth muscle cells in atherosclerosis. Circ Res. 2016;118(4):692-702. https://doi.org/10.1161/ CIRCRESAHA.115.306361

\section{Publisher's Note}

Springer Nature remains neutral with regard to jurisdictional claims in published maps and institutional affiliations. 